\title{
Evaluating LCOE in sustainable microgrids for smart city applications
}

\author{
Stefano Bracco ${ }^{1}$, Federico Delfino ${ }^{1}$, Paola Laiolo $^{1}$, Luisa Pagnini ${ }^{2 *}$, and Giorgio Piazza ${ }^{1}$ \\ ${ }^{1}$ University of Genoa, DITEN (Electrical, Electronics and Telecommunication Engineering and Naval \\ Architecture Department), Via all’Opera Pia 11a, 16145 Genoa, Italy \\ ${ }^{2}$ University of Genoa, DICCA (Department of Civil, Chemical and Environmental Engineering), Via \\ Montallegro 1, 16145 Genoa, Italy
}

\begin{abstract}
A microgrid can be considered a profitable solution to be adopted in smart cities if it is marketable, i.e. more, or at least equally convenient than other traditional energy supply sources. Different economic parameters can be defined to determine its affordability. In particular, the LCOE (Levelized Cost of Electricity) is the most popular indicator adopted in the energy sector, widely used both for conventional and renewable power sources. However, the use of this metric still disregards important aspects that concerns microgrid applications. After providing a state-of-the-art of the use of LCOE, the present paper proposes a new methodology for sustainable microgrids in smart city, taking into account benefits due to cogeneration and trigeneration, integration costs as well as positive and negative side effects.
\end{abstract}

\section{Introduction}

In the last decade, in the energy sector, the attention has been more and more devoted to the exploitation of renewable sources and to smart grid and microgrid applications [1, 2]. Consequently, the power system scenario has changed significantly and, nowadays, several low and medium size dispersed generation units and storage systems are connected to medium and low voltage electrical networks [3].

The regeneration of urban areas is often based on the design and the installation of polygeneration microgrid systems which are usually characterized by the presence of renewable source power units (mainly based on the exploitation of solar, wind and geothermal energy), cogeneration and trigeneration systems (usually internal combustion engines or microturbines coupled with absorption chillers and heat pumps), thermal and electrical storage. Such sustainable microgrids are seen as cost-effective, environmentalfriendly technical solutions able to satisfy the energy needs of different users, providing services to Distribution System Operators.

In microgrids, storage systems are mainly used to better exploit renewable sources and other dispatchable units, to smooth load peaks and to reduce the amount of energy absorbed from the electrical grid. They can be managed to optimize the operation of combined heat

\footnotetext{
* Corresponding author: luisa.pagnini@unige.it
} 
and power (CHP) units and to limit the use of traditional boilers, thus reducing carbon dioxide emissions and allowing the CHP operation at maximum efficiency. Therefore, many advantages derive from the application of the microgrid concept in the urban environment and, in the near future, microgrids will represent one of the main pillars of sustainable smart cities.

The LCOE is a popular indicator used in the energy sector. Referring to a power plant, it can be calculated as the ratio between the costs (capital and operating) of the plant and the generated amount of energy throughout the life cycle. It supplies a simple procedure to compare the competitiveness of different electricity generating systems. As an example, refs [4-10] supply technical surveys in terms of LCOE for different generating sources in different countries, evaluating the economic competitiveness in the electricity market. On the other hand, this metric is strictly related to the quantities accounted for, the model used, and the assumptions made. It may therefore give rise to incomplete or misleading evaluations.

The application to conventional power plants integrated with renewable production, such as photovoltaic (PV) plants and wind turbines, should address the intermittent nature of the source, as uncertainties in its forecast can significantly influence the LCOE values. Tran et al. [11] highlight that, unlike the well-established traditional energy technologies, renewable energy production is subject to high uncertainties in terms of costs. Ueckerdt et al. [12] focus on the issue of integration costs to be added to the direct costs of wind and solar power and introduce the so-called System LCOE which, for each considered technology, considers the sum of generation and integration costs.

In CHP plants, the cost assessment should carefully take into account a number of factors depending on the chosen technology and on the market price scenarios. Hennessy et al. [13] highlight the importance of these aspects referring to a given typology of heat source. Mundada et al. [14] provide a method to quantify the LCOE of PV, battery and CHP hybrid systems, however, they do not include the additional value of the heat. Nian et al. [15] adapt the LCOE model to evaluate the Levelized Cost of Heat, considering the conversion efficiency of electricity and heat production, and showing the profitability of cogeneration.

The adoption of energy storage is a viable solution, even if still costly, especially when associated to renewable energy production. The Lazard study provides an analysis of the most important storage technologies by using LCOS - Levelized Cost of Storage [10]. Obi et al. [16] propose an advanced algorithm and compare different storage systems. Lai and McCulloch [17] propose a specific indicator called Levelized Cost of Delivery that generalises the LCOE metric to the electrical energy storage.

The integration cost of the different generation technologies represents an important issue for LCOE evaluation of microgrids. Lofti and Khoadei [18] derive the LCOE of a microgrid by summing the contributions of each energy resource; in this case LCOE assumes an intermediate value between the lowest and the larger LCOE of each considered technology. However, they do not consider integration costs and externalities.

In addition to all the above mentioned issues, a number of other aspects must be considered, whose costs are difficult to be quantified. A comprehensive evaluation should address the economic and environmental externalities [19], [20] that have a significant effect on the viability of the different generating technologies [21]. Considering air pollution and impact on human health, the LCOE of renewable power sources is likely to decrease; on the other hand, noise, land usage and presence of transmission lines may negatively affect the total cost of renewable energy. Many infrastructures such as campuses and other social facilities that include renewable and other power sources integrated in microgrids are funded via research grants; also the social acceptance produces medium and long-term benefits that increase the value of the infrastructure. Similar advantages may 
derive for the Utility owning and managing the microgrid, that can benefit from a positive reputation effect. Despite this positive feedback, LCOE models present in literature still ignore factors related to Environmental, Social and Governance (referred to as ESG).

Starting from these premises, the present paper provides an advanced LCOE formulation for microgrids with renewable distributed power sources, CHP units and storage, accounting both for integration costs and cogeneration benefits. Externalities and positive side effects that come from sustainable energy production are quantified introducing a new indicator called Levelized Cost of Demand - LCOD.

\section{The methodology}

A methodology is here presented to evaluate the LCOE of a microgrid providing electrical, thermal and cooling energy to a urban district (e.g., a university campus, a military compound or a pavilion hospital) characterized by a single point of common coupling with the public distribution grid. The considered installed technologies are PV, cogeneration gas microturbines (mGT), absorption chillers (AC) and electrical storage batteries (STO). The electrical demand of the site is satisfied by the PV, the mGT, the STO and the public grid, whereas the thermal demand (heating and domestic hot water) is provided by mGT. The cooling demand is satisfied by the AC driven by mGT.

For each of the aforesaid technologies a suitable economic indicator can be calculated, specifically LCOE for the PV and for the mGT+AC trigeneration plant and LCOS for STO. The LCOE of the PV plant can be computed as:

$$
\operatorname{LCOE}^{P V}=\frac{\sum_{j=0}^{N^{P V}}\left[\frac{I_{j}^{P V}+C_{j}^{P V}}{(1+r)^{j}}\right]}{\sum_{j=0}^{N^{P V}}\left[\frac{E_{j}^{P V}}{(1+r)^{j}}\right]}
$$

where $I_{j}^{P V}$ and $C_{j}^{P V}$ indicate respectively the capital and operating costs at year $j(j=0$ is the year of commissioning, $j=N^{P V}$ refers to the end of useful life); operating costs typically refer to maintenance; $E_{j}^{P V}$ is the annual electricity production and $r$ is the discount rate.

For the trigeneration plant (TP), composed of the microturbines and the absorption chillers, the LCOE can be defined as:

$$
L C O E^{T P}=\frac{\sum_{j=0}^{N^{T P}}\left[\frac{I_{j}^{m G T}+I_{j}^{A C}+C_{j}^{m G T}+C_{j}^{A C}-B_{j}^{H}-B_{j}^{C}}{(1+r)^{j}}\right]}{\sum_{j=0}^{N^{T P}}\left[\frac{E_{j}^{m G T}}{(1+r)^{j}}\right]}
$$

where $I_{j}^{m G T}$ and $I_{j}^{A C}$ indicate respectively the capital cost of $\mathrm{mGT}$ and AC at year $j$; $C_{j}^{m G T}$ and $C_{j}^{A C}$ represent operating costs, that take into account maintenance costs and expenses for fuel feeding mGT; $E_{j}^{m G T}$ is the annual electricity production of mGT and $N^{T P}$ is the useful life of the trigeneration plant; $B_{j}^{H}$ and $B_{j}^{C}$ are avoided expenses. $B_{j}^{H}$ quantifies the economic benefit due to the heat $(\mathrm{H})$ production, $B_{j}^{C}$ quantifies the benefit of the cooling $(\mathrm{C})$ energy production. These two terms account for the expenses that should be 
incurred if traditional systems (such as gas boilers and compression chillers) were used to satisfy thermal and cooling loads. Consequently, in Eq. (2) they are subtracted from the operating costs.

For the storage system, the LCOS can be computed as:

$$
L C O S=\frac{\sum_{j=0}^{N^{S T O}}\left[\frac{I_{j}^{S T O}+C_{j}^{S T O}}{(1+r)^{j}}\right]}{\sum_{j=0}^{N^{S T O}}\left[\frac{E_{j}^{\text {out }} \text { STO }}{(1+r)^{j}}\right]}
$$

where $I_{j}^{S T O}$ and $C_{j}^{S T O}$ indicate respectively the capital and maintenance cost of storage batteries, $E_{j}^{\text {out }}$ STO is the energy released by the storage and $N^{\text {STO }}$ is the useful life of batteries. No charging costs are considered since the storage system is charged by PV ad $\mathrm{mGT}$, and therefore these costs are already computed in $L C O E^{P V}$ and $L C O E^{T P}$.

The system LCOE for the microgrid is calculated as:

$$
\operatorname{LCOE}_{\mu g}=\frac{\sum_{j=0}^{N}\left[\frac{I_{j}^{p}+I_{j}^{\mu g}+C_{j}^{p}-B_{j}}{(1+r)^{j}}\right]}{\sum_{j=0}^{N}\left[\frac{E_{j}^{\text {local }}}{(1+r)^{j}}\right]}
$$

where $E_{j}^{\text {local }}$ is the electricity local production (from PV and mGT), $I_{j}^{p}$ and $C_{j}^{p}$ indicate the sum of capital and operating costs of technologies (PV, TP, STO), $I_{j}^{\mu g}$ is the sum of integration costs (e.g., cables, pipelines, ICT), $B_{j}=B_{j}^{H}+B_{j}^{C}$ represents the benefit related to cogeneration and trigeneration, $N$ is the lifespan of the infrastructure.

The supply cost for the User of the microgrid should consider all the aforementioned costs, the extenalities, such as emission of pollutants, soil occupation, noise pollution, as well as social and economic benefits derived from the application of the sustainable microgrid concept. Therefore, a new indicator called Levelized Cost of the Demand (LCOD) is defined as the ratio between the sum of discounted costs and the sum of discounted energy demands of the site:

$$
L C O D=\frac{\sum_{j=0}^{N}\left[\frac{I_{j}+C_{j}-B_{j}+X_{j}}{(1+r)^{j}}\right]}{\sum_{j=0}^{N}\left[\frac{D_{j}}{(1+r)^{j}}\right]}
$$

where $D_{j}$ is the annual electricity demand, $I_{j}$ and $C_{j}$ are the total capital and operating costs, respectively, whereas $X_{j}$ accounts for the generalized externalities, herein considered either as costs (positive) or as benefits (negative).

\section{Results}

All the previous concepts and equations are applied to a microgrid feeding a university campus located in the north of Italy, with laboratories, sport facilities and a swimming pool 
that is covered and heated during the winter and uncovered during the summer season. The campus also has student housings and areas rent to private companies, making this site very similar to a urban district. All the facilities are characterized by the annual electricity, thermal and cooling energy demand reported in Table 1 (second column). In the 'as-is' configuration, the satisfaction of the thermal, cooling and electricity demand is obtained, respectively, by boilers fed by natural gas (with efficiency $\eta=0.85$ ), by compression chillers (with Energy Efficiency Ratio EER=2.8) and by the national grid, since no microgrid is installed. The 'to-be' scenario considers the dismission of boilers and compression chillers and the installation of a microgrid, composed of a PV plant, two microturbines coupled with two absorption chillers and an electrical storage (third column in Table 1). The benefits of positive side effects are the profits from research contracts, fundings obtained thanks to the innovative character of the microgrid and to higher appeal on students.

The prices of natural gas and electricity, as well as the investment and operating costs of each technology, are assumed according to three different price levels (Low, Medium and High), accounting for fluctuations on the market. Table 2 reports the investment costs for each technology of the microgrid for the three considered levels.

Table 1. As-is versus to-be scenario

\begin{tabular}{|c|c|c|}
\hline & as-is & to-be \\
\hline Electric demand [MWhel] & 1778.5 & 1600 \\
\hline Thermal demand [MWhth] & 1734 & 1734 \\
\hline Cooling Demand [MWh $\left.\mathrm{h}_{\mathrm{co}}\right]$ & 499.8 & 499.8 \\
\hline Electricity supply & public grid & PV (120 kW peak), mGT (2x100 kWel), public grid \\
\hline Thermal energy supply & boilers $(\eta=0,85)$ & mGT $(2 \times 170 \mathrm{~kW}$ th $)$ \\
\hline Cooling energy supply & $\begin{array}{c}\text { compression } \\
\text { chillers }(\mathrm{EER}=2.8)\end{array}$ & AC $(2 \times 119$ kW $\mathrm{co})$ \\
\hline Electric storage & no & STO (150 kWh) \\
\hline
\end{tabular}

Table 2. Capital costs of technologies

\begin{tabular}{l|c|c|c} 
& Low & Medium & High \\
\hline$I^{P V}\left[€ / \mathrm{kW}_{\text {peak }}\right]$ & 1000 & 1250 & 1500 \\
\hline$I^{m G T}\left[€ / \mathrm{kW}_{\mathrm{el}}\right]$ & 1000 & 1500 & 2000 \\
\hline$I^{A C}[€ / \mathrm{kW}$ co $]$ & 900 & 1000 & 1100 \\
\hline$I^{S T O}[€ / \mathrm{kWh}]$ & 400 & 550 & 700
\end{tabular}

Figure 1a shows some results of the application of Eq. (4) to the microgrid (i.e., scenario 'to-be'), considering different discount rates. In all cases, LCOE values are lower than the price of the electricity purchased from the national grid (Low: $0.14 € / \mathrm{kWh}$, Medium: $0.16 € / \mathrm{kWh}$, High: $0.18 € / \mathrm{kWh})$. For the Medium case, Figure $1 \mathrm{~b}$ reports LCOD values for the two scenarios and proves that the microgrid installation can be considered a winning choice also from the user perspective.

\section{Conclusions}

The present paper has outlined a comprehensive methodology for the assessment of the levelized cost of electricity in smart city applications, considering both conventional and integrated renewable power sources and including the benefits of cogeneration and other side effects. A preliminary application highlights the economical feasibility of the 
regeneration of a urban district by the installation of a sustainable polygeneration microgrid.
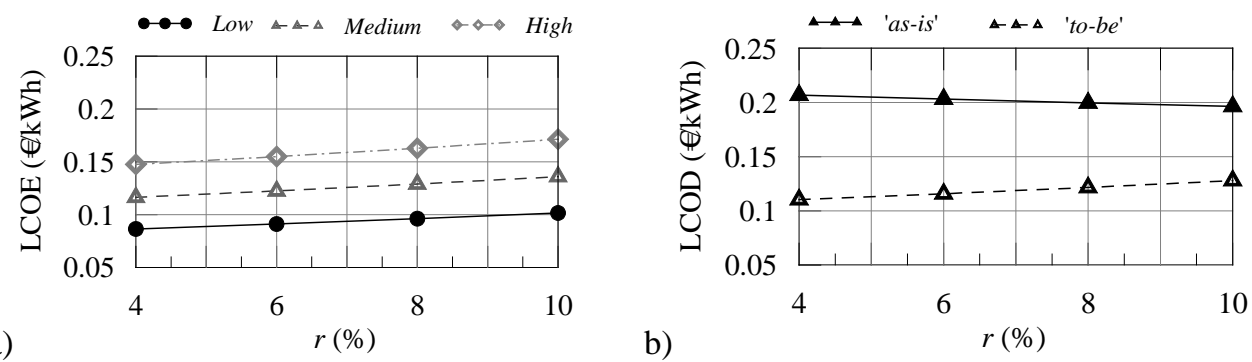

Fig. 1. LCOE and LCOD of the investigated scenarios

\section{References}

1. F. Delfino, R. Procopio, M. Rossi, S. Bracco, M. Brignone, Microgrid Design and Operation: Toward Smart Energy in Cities (Artech House, 2018)

2. M.S. Hossain, N.A. Madlool, N.A. Rahim, J. Selvaraj, A.K. Pandey, A.F. Khan, Renew Sustain Energy Rev 60 1168-1184 (2016)

3. S. Bracco, F. Delfino, G. Ferro, L.C. Pagnini, M. Robba, M. Rossi, Appl Energy, 228 2288-2297 (2018)

4. Energia elettrica, anatomia dei costi, Ricerca sul Sistema Energetico (RSE SpA, Alkes, 2014)

5. M. Rao, C. Tricoli. Indicatori di costo nella generazione energetica, analisi critica del concetto di LCOE (ENEA, 2015)

6. M.L. Guillerminet, D. Marchal, R. Gerson, Y. Berrou, Y. Coûts des énergies renouvelables en France (ADEME, Cedex, 2016)

7. S.D. Commello, G. Glenk, S. Reichelstein, Levelized Cost of Electricity Calculator: A User Guide (Stanford Graduate School of Business, 2017)

8. C. Kost, S. Shammugan, V. Julch, H.T. Nguyen, T. Schlegl, T. Levelized cost of electricity, Renewable Energy Technology (Fraunhofer Institutefor Solar Energy Systems, Freiburg 2018).

9. Lazard's levelized cost of energy analysis (Lazard, 2018)

10. Lazard's levelized cost of storage analysis (Lazard, 2018)

11. T.T.D. Tran, T.D. Smith. Incorporating performance-based global sensitivity and uncertainty analysis into LCOE calculations for emerging renewable energy technologies, Appl Energy 216 157-171(2018)

12. F. Ueckerdt, L. Hirth, Energy, 63 61-75(2013)

13. J. Hennessy, H. Li, F. Wallin, E. Thorin, Appl Energy, 228 766-776 (2018)

14. A.S. Mundada, K.K. Shah, J.M. Pearce, Renew Sustain Energy Rev 57 692-703 (2016)

15. V. Nian, Q.S.Z. Ma, H. Li, Energy Procedia 104556 - 561 (2016)

16. M. Obi, S.M. Jensen, J.B. Ferris, R.B. Bass, Renew Sustain Energy Rev 67 (2017) 908-920

17. C.S. Lai, M.D.A. McCulloch, Appl Energy 190 191-203 (2017)

18. H. Lotfi, A Khodaei, A. IEEE Power Eng Soc 7741379 (2016)

19. K.J. Benes, C. Augustin, The Electricity Journal 29 48-54 (2016)

20. J.D. Rhodes, C. King, et al, Energ Policy 102 441-449 (2017)

21. I.F. Roth, L. Ambs, Energy 29 2125-2144 (2004) 